\title{
CLASSIFICAÇÃO DE PESSOAS NA PROVA TUBERCULINICA: APLICAÇÃO DE UM MODELO ESTATISTICO QUANDO A DISTRIBUIÇÃO DE FREQỦENCIAS DA INDURAÇÃO É UMA MISTURA DE COMPONENTES NORMAIS
}

Sanches, O. - Classificação de pessoas na prova tuberculinica: aplicação de um modelo estatístico quando a distribuição de freqüências da induração é uma mistura de componentes normais. Rev. Saúde públ., S. Paulo, 10: $285-9,1976$.

Resumo: O problema de classificar pessoas de acordo com o tamanho da induração, na prova tuberculinica segundo a técnica de Mantoux, é resolvido, para um conjunto de dados obtidos em uma população genérica, utilizando-se o critério estatistico de "melhores regiōes possiveis de classificação". São obtidas estimativas das probabilidades de classificacão errada.

UNitermos: Teste tuberculinico. Induração. Mistura de distribuições normais. Regióes de classificação. Probabilidade de classificação errada.

\section{INTRODUC ÃO}

Em publicação anterior, Sanches ${ }^{4}$ (1975), utilizando um método gráfico aproximado, discutiu-se a possibilidade de se decompor, em componentes normais, uma distribuição de freqüências de medidas de induração ou de eritema, na prova tuberculínica segundo a técnica de Mantoux, obtidas em uma população genérica, objetivando a posterior solução do problema de classificação segundo o tamanho da reação.

O objetivo do presente trabalho é uma complementação do anterior, isto é, utilizando os resultados anteriormente obtidos. relativamente à variável induração, mostrar que é possivel estabelecer esti- mativas das regiões de classificação para cada um dos grupos componentes, segundo um modelo estatístico estabelecido na literatura, assim como determinar as estimativas das probabilidades de classificação errada.

2. CONSIDERAÇOES SOBRE O PROBLEMA DE CLASSIFICAÇAO EM UM ENTRE $k$ GRUPOS COMPONENTES DE UMA MISTURA DE DISTRIBUIÇOES NORMAIS

O que segue tem a finalidade de colocar o leitor interessado, não especialista em Estatística, em contacto com o modelo de classificação a ser utilizado. O assunto, no entanto. é discutido de forma ampla e exaustiva em quaisquer textos de análise multivariada.

* Da Escola de Enfermagem de Ribeirão Preto da USP -.. Campus de Ribeirão Preto, SP Brasil. 
SANCHES, O. - Classificação de pessoas na prova tuberculínica: aplicação de um modelo estatístico quando a distribuição de frequências da induração é uma mistura de componentes normais. Rev. Saude públ., S. Paulo, 10:285-9, 1976.

Consideremos uma mistura de $\mathrm{k}$ distribuções normais, univariadas, de médias $\mu_{i}$ e variâncias $\sigma_{i}^{2}$, sendo $p_{i}$ as proporçôes da mistura, $\mathrm{i}=1,2, \quad ., \mathrm{k}$.

$O$ problema geral consiste em classificar um elemento desta mistura, retirado ao acaso. como pertencente a um dos $\mathrm{k}$ grupos componentes. Nas condições consideradas, cada elemento é representado por um ponto de um espaço unidimensional. Assim, o problema de classificação é equivalente ao de dividir o espaço unidimensional referido em $k$ regiões $R_{1}, R_{2}$, ., $\mathrm{R}_{\mathrm{k}}$, mutuamente exclusivas, de tal modo que permita colocar no $i^{\text {mo }}$ grupo componente, um elemento representado por um ponto de $R_{i}, i=1,2, \quad ., k$.

Se um elemento do $\mathrm{i}^{\text {mo }}$ grupo tem uma probabilidade $\beta_{i}$ de pertencer a $R_{i}$, então o valor esperado da proporção de classificações erradas é:

$$
\alpha=1-\sum_{i=1}^{k} p_{i} \beta_{1}
$$

A questão é escolher as regiões de tal modo que $\alpha$ seja mínima, isto é,

$$
\sum_{i=1}^{k} p_{1} \beta_{1} \text { seja máxima. }
$$

Tais regiōes, quando existem, são denominadas "melhores regióes possíveis de classificação". Rao ${ }^{2}$ (1952) e Anderson $^{1}$ (1958) demonstram que regiónes definidas por:

$$
\begin{aligned}
& \mathrm{R}_{\mathrm{i}}=\left\{\mathrm{x} / \mathrm{p}_{\mathrm{i}} \mathrm{f}_{\mathrm{i}}(\mathrm{x}) \geq \mathrm{p}_{\mathrm{j}} \mathrm{f}_{\mathrm{j}}(\mathrm{x})\right. \\
& \mathrm{i} \neq \mathrm{j} ; \mathrm{i}, \mathrm{j}=1,2, \quad, \mathrm{k}\}
\end{aligned}
$$

satisfazem o critério de melhores regiōes possiveis $\left(f_{i}(x)\right.$ é a função densidade de probabilidade da $i^{\text {ma }}$ componente da mistura).

Para o caso em consideração as desigualdades contidas em (2.1) podem ser escritas, explicitamente:

$$
\begin{gathered}
\left(\sigma_{j}^{2}-\sigma_{i}^{2}\right) \mathrm{x}^{2}-2\left(\sigma_{j}^{2} \mu_{i}-\sigma_{i}^{2} \mu_{j}\right) x \\
+\left[\sigma_{j}^{2} \mu_{i}^{2}-\sigma_{i}^{2} \mu_{j}^{2}-\sigma_{i}^{2} \sigma_{j}^{2}\right.
\end{gathered}
$$

$$
\begin{aligned}
& \left.\log \left(\frac{p_{1} \sigma_{j}}{p_{j} \sigma_{1}}\right)^{2}\right] \leq 0 \\
& i \neq j ; i, j=1,2, \quad ., k .
\end{aligned}
$$

Se uma ou mais componentes são truncadas, é suficiente introduzir, em (2.1), a correção para o truncamento. Assim, apenas para fixar idéias, suponhamos a $\mathrm{i}^{\text {ma }}$ componente truncada, à esquerda, no ponto $\xi_{11}$, suposto conhecido. Nestas condiçôes a desigualdade (2.2) se escreve:

$$
\begin{gathered}
\left(\sigma_{j}^{2}-\sigma_{i}^{2}\right) x^{2}-2\left(\sigma_{j}^{2} \mu_{i}-\sigma_{i}^{2} \mu_{j}\right) \\
x+\left[\sigma_{j}^{2} \mu_{i}^{2}-\sigma_{i}^{2} \mu_{j}^{2}-\sigma_{i}^{2} \sigma_{j}^{2} \log \right. \\
\left(\frac{K_{i e} p_{i} \sigma_{j}}{p_{j} \sigma_{i}}\right)^{2} \leq 0
\end{gathered}
$$

onde $\mathbf{K}_{\mathrm{i} e}$ é o fator de correção para o truncamento,

$$
i \neq j ; i, j=1,2, \quad ., k \text {. }
$$

Se $\mu_{i}^{*}, \sigma_{i}^{2}$ e $p_{i}^{*}$ são estimativas de $\mu_{i}, \sigma_{i}^{2}$ e $p_{i}$, a sua substituição em (2.2) ou $(2.2 \mathrm{a})$ fornece estimativas $\mathrm{R}_{\mathrm{i}}^{*}$ de $\mathbf{R}_{\mathrm{i}}$; $\mathrm{i}=1,2, \quad ., \mathrm{k}$.

As probabilidades de classificar erradamente como pertencentes ao $\mathrm{i}^{\text {mo }}$ grupo elementos pertencentes aos $(k-1)$ grupos restantes são dadas por:

$$
\begin{array}{r}
\alpha_{i j}=\int_{R_{1}} p_{j} f_{j}(x) d x ; \\
i \neq j ; i, j=1,2, \quad ., k
\end{array}
$$

as quais são estimadas por:

$$
\alpha_{i j}^{*}=\int_{R_{i}^{*}} p_{j}^{*} f_{j}^{*}(x) d x
$$

sendo $f_{j}^{*}(x)$ uma estimativa de $f_{j}(x)$. 
SANCHES, O. - Classificação de pessoas na prova tuberculínica: aplicação de um modelo estatístico quando a distribuição de frequiências da induração é uma mistura de componentes normais. Rev. Saúde públ., S. Paulo, 10:285-9, 1976.

3. CLASSIFICAÇAO DE PESSOAS, EM UMA POPULACÃO GENERICA, SEGUNDO DIAMETROS DA INDURACAO NA PROVA TUBERCULINICA

Utilizando-nos de dados obtidos por Ruffino Netto ${ }^{3}$ (1970), Sanches ${ }^{4}$ (1975) mostra que a distribuição de frequiências para as medidas de induração podiam ser decompostas em três grupos componentes, com distribuições normais, sendo a primeira componente, por decisão do autor, truncada à esquerda no ponto correspondente à medida de $2 \mathrm{~mm}$.

A Tabela 1 apresenta a distribuição de freqüências observadas $e$ as distribuiçóes componentes normais esperadas, referentes às medidas de diâmetros de induração, estudadas no trabalho acima citado, enquanto que a Tabela 2 apresenta as estimativas dos parâmetros obtidas para tais componentes.

T A B E A 1

Medldas de induração na prova tuberculínica, segundo a técnica de Mantoux: distribuição de freqüências observada e distribuiçōes componentes esperadas.

\begin{tabular}{|c|c|c|c|c|}
\hline \multirow{2}{*}{$\begin{array}{l}\text { Induração } \\
(\mathrm{mm})\end{array}$} & \multirow{2}{*}{$\begin{array}{l}\text { Fre- } \\
\text { qüências } \\
\text { obser- } \\
\text { vadas }\end{array}$} & \multicolumn{3}{|c|}{$\begin{array}{c}\text { Freqüências } \\
\text { esperadas }\end{array}$} \\
\hline & & $\begin{array}{c}1.8 \\
\text { comp. }\end{array}$ & $\begin{array}{c}2 \mathrm{~s} \\
\text { comp. }\end{array}$ & $\begin{array}{c}3 . \\
\text { comp. }\end{array}$ \\
\hline $21-4$ & 253 & 251 & 1 & 2 \\
\hline $41-6$ & 108 & 93 & 11 & 4 \\
\hline $61-8$ & 37 & 1 & 26 & 10 \\
\hline $8 i-10$ & 39 & & 25 & 20 \\
\hline $10 \mid-12$ & 30 & & 5 & 27 \\
\hline $12-14$ & 24 & & & 26 \\
\hline $14-16$ & 16 & & & 18 \\
\hline $16-18$ & 8 & & & 9 \\
\hline $18 \mid-20$ & 2 & & & 3 \\
\hline Total & 517 & 345 & 68 & 119 \\
\hline
\end{tabular}

Fonte: Sanches' (1975).

\section{TA B E A 2}

Estimativas dos parâmetros das três componentes esperadas, referidas na tabela 1

\begin{tabular}{l|rc|c}
\hline \multirow{2}{*}{ Componente } & \multicolumn{3}{|c}{ Estimativas } \\
\cline { 2 - 4 } & $\begin{array}{c}\mu^{*} \\
(\mathrm{~mm})\end{array}$ & $\begin{array}{c}\sigma^{*} \\
(\mathrm{~mm})\end{array}$ & $\begin{array}{c}\mathbf{p}^{*} \\
(\%)\end{array}$ \\
\hline Primeira & $\mathbf{3 , 5 3}$ & 0,94 & 65,34 \\
Segunda & 7,60 & 1,79 & 12,11 \\
Terceira & 11,80 & 3,43 & 22,54 \\
\hline
\end{tabular}

De (2.2) e (2.2a), utilizando os dados da Tabela 2, obtemos as estimativas para as regiốes de classificação dos três grupos componentes:

$$
\begin{gathered}
R_{1}^{*}=\{x / x<5,27 \mathrm{~mm}\} \\
R_{2}^{*}=\{x / 5,27 \mathrm{~mm} \leq x<8,98 \mathrm{~mm}\} \\
R_{3}^{*}=\{x / 8,98 \mathrm{~mm}<x\}
\end{gathered}
$$

Com tais estimativas, a partir de (2.3a), utilizando-nos de uma tabela da $N(0 ; 1)$ obtemos as estimativas das probabilidades de classificação errada:

$$
\begin{aligned}
& \alpha_{12}^{*}=0,0116 \text { ou } 1,16 \% \\
& \alpha_{13}^{*}=0,0060 \text { ou } 0,60 \% \\
& \alpha_{21}^{*}=0,0222 \text { ou } 2,22 \% \\
& \alpha_{23}^{*}=0,0400 \text { ou } 4,00 \% \\
& \alpha_{31}^{*}=0,0000 \\
& \alpha_{32}^{*}=0,0267 \text { ou } 2,67 \%
\end{aligned}
$$

\section{Discussão e CONCLUSÃo}

Se uma distribuição de freqüências observada, de medidas de induração na 
SANCHES, O. - Classificação de pesscas na prova tuberculínica: aplicação de um modelo estatístico quando a distribuição de frequêencias da induração é uma mistura de componentes normais. Rev. Saúde públ., S. Paulo, 10:285-9, 1976.

prova tuberculínica, mostra-se decomponível em componentes normais, é possível a aplicação do critério estatístico de "melhores regiões possiveis" para estabelecer estimativas das regiões de classificação das pessoas pertencentes aos distintos grupos componentes, assim como estimativas das probabilidades de classificação errada.

Isto é interessante pois que o citado critério leva em consideração as proporçóes com que cada grupo entra na inistura. Ora, se estas proporções podem variar em função de alguns fatores como área geográfica considerada, grupos etários considerados, raça etc., há interesse buscar, em função de tais fatores, as regiōes de classificação e estudá-las, em diferentes épocas, para se aquilatar de possíveis modificações nos seus valores.

A possibilidade do cálculo das estimativas das probabilidades de classificação errada permite ao pesquisador decidir se o critério estatístico de "melhores regiōes possiveis" atendam ou não aos objetivos médicos.

Assim. com os dados utilizados, as maiores probabilidades de classificação errada foram:

o (! ${ }_{23}^{*}=0,0400$, isto é, a probabilidade de classificar, erradamente, como pertencente ao segundo grupo, um elemento do terceiro grupo é de 4 em 100.

ii) $\alpha_{32}^{*}=0.0267$, isto é. a probabilidade de classificar. erradamente, como pertencente ao terceiro grupo, um elemento pertencente ao segundo grupo é de, aproximadamente, 3 em 100 .

iii) $\alpha_{21}^{*}=0.0222$, isto é, a probabilidade de classificar, erradamente, como pertencente ao segundo grupo. um ele- mento do primeiro grupo é de, aproximadamente, 2 em 100.

Se tais estimativas forem consideradas elevadas, segundo os objetivos do pesquisador, é possivel fixar uma probabilidade de classificação errada tão pequena quanto se deseje e, em função desta probabilidade pré-fixada, determinar novas regiōes de classificação tentando diminuir, é claro, o custo da classificação errada. Procedimentos deste tipo são discutidos, por exemplo, em Rao ${ }^{2}$ (1952), os quais, no entanto, não mais satisfazem o critério estatístico de "melhores regiōes possiveis".

Finalmente, é interessante confrotar os resultados por nós obtidos com aqueles estabelecidos pela Comissão Nacional contra a Tuberculose. Esta, em sua $2 .^{a}$ Recomendação (1968), considerando a dose de 2 T.U de $R_{t} 23$, estahelece que "até que seja possível fixar o exato tamanho em milímetros, acima e abaixo do qual devam as reações ser consideradas específicas (positivas) e inespecíficas (negativas) deve-se adotar o seguinte critério de interpretação dos resultados da prova Tuberculínica Padronizada:

$$
\begin{array}{lc}
\text { Não reatores: } & 0-4 \mathrm{~mm} \\
\text { Reatores fracos: } & 5-9 \mathrm{~mm} \\
\text { Reatores fortes: } & 10 \mathrm{~mm} \text { ou mais }
\end{array}
$$

Utilizando a nomenclatura acima, e arredondando-se para o número inteiro de milímetros mais próximo, o critério de classificação por nós utilizado, para os dados considerados, permite a seguinte classificação:

Não reatores: menores que $5 \mathrm{~mm}$

Reatores fracos: de 5 a $8 \mathrm{~mm}$ inclusives

Reatores fortes: $9 \mathrm{~mm}$ ou mais 
SANCHES, O. - Classificação de pessoas na prova tuberculinica: aplicação de um modelo estatístico quando a distribuição de frequencıs da induracão é uma mistura de componentes normais. Rer. Sulle pribt., S. Paulo. 10:285-9, 1976.

RSPU-B $/ 324$

SANCHES, O. - [Classification of subjects in the tuberculin test: application of a statistical model when the frequency distribution of the induration is a mixture of nomal components]. Rev. Saúde púbI., S. Paulo, 10:285-9.9.

SumMary: The classification problem in the tubercutin test uas studied. A sample of induration data from a generic population was used and the "best possible regions" criterion was applied. Estimates of classification regions and estimates of probabilities of misclassification were obtained.

UNITERMs: Tuberculin test. Induration. Mixture of nomal distributions. Classification regions. Misclassification mrobability.

\section{REFERENCIAS BIBLIOGRAFICAS}

1. ANDERSON. T. W-An introduction to multivariate statistical analysis. New York, John Wlley \& Sons, 1958.

2. RAO, C. R. - Advanced statistical methods in biometric research. New Fork, John Wiley \& Sons, 1952.

3. RLFFINo NetTo, A. - Epidemiologia Ia tuberculose: estudo de alguns aspertos mensurivers na prova tuberculincer. Piberão Preto, 1970. [Te- se de Doutoramento - Faculdade de Medicina de Ribeirão Preto da USPJ.

4. SANCHES, O. - Distribuicão das medidas de induracão e eritema na prova tuberculinica: aplicacão de um métocio gráfico de decomposicão de uma distribuicão de frequências em componentes normais. Rer. Saude publ., S. Paulo, 9:15-24, 1975 .

Recebido para publicario em 22/03/1976

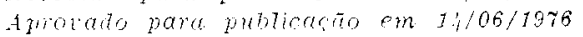

